\title{
Efeito do vapor d'água na síntese pelo método do precursor polimérico da alumina contendo aditivos
}

\section{(Effect of water vapor on the synthesis by the polymeric precursor method of alumina doped powders)}

\author{
R. H. R. Castro, D. Gouvêa \\ Departamento de Engenharia Metalúrgica e de Materiais, Escola Politécnica, USP \\ Av. Prof. Mello Moraes, 2463, Cidade Universitária, S. Paulo, SP 05508-900
}

\begin{abstract}
Resumo
A presença de $\mathrm{H}_{2} \mathrm{O}$ nos processos de síntese e transformação de fase da alumina tem usualmente função catalisadora, diminuindo as temperaturas dos processos e facilitando os possíveis rearranjos atômicos. Neste trabalho mostrou-se que o vapor de $\mathrm{H}_{2} \mathrm{O}$ durante a síntese pode não apenas acelerar os processos diminuindo as energias de ativação, mas também induzir diferentes formas de ação de aditivos. O estudo foi possível utilizando-se o método dos precursores poliméricos para a síntese dos pós, que permite um controle absoluto do teor de $\mathrm{H}_{2} \mathrm{O}$ na etapa de cristalização do material. Alumina contendo $\mathrm{Mn}$ ou $\mathrm{Mg}$ como aditivos foram sintetizadas na ausência de $\mathrm{H}_{2} \mathrm{O}$ e na presença de excesso controlado desta e fases diferentes para cada situação foram observadas. Uma explicação do fenômeno é descrita em função da dependência da cristalização da $\gamma-\mathrm{Al}_{2} \mathrm{O}_{3}$ na presença de $\mathrm{H}^{+}$para compensação das vacâncias catiônicas.
\end{abstract}

Palavras-chave: gama-alumina, aditivos.

\begin{abstract}
It is commonly accepted that the presence of $\mathrm{H}_{2} \mathrm{O}$ during the synthesis and phase transformation of alumina causes decreasing temperatures of these processes since it increases the atomic motilities. In addition to this general concept, in this work $\mathrm{H}_{2} \mathrm{O}$ vapor is also shown to play an important role on the influence of additives in alumina based systems. Alumina powders containing Mn or $\mathrm{Mg}$ were synthesized by the polymeric precursor method under $\mathrm{H}_{2} \mathrm{O}$ vapor or dry atmosphere during the crystallization stage. Each atmosphere promoted a different phase and an explanation for the phenomenon was given bases on the $\gamma$-Al $\mathrm{O}_{3}$ crystallization dependence on the $H^{+}$presence due to the required cationic vacancies compensations.
\end{abstract}

Keywords: gamma-alumina, additives.

\section{INTRODUÇÃO}

Atualmente o método de produção de alumina a partir de um precursor polimérico [1,2], baseado na patente de Pechini $[3,4]$, tem demonstrado bastante eficácia na preparação de pós com alta homogeneidade e reatividade química. Sua vantagem está principalmente ligada à nucleação múltipla e isolada durante a pirólise do polímero e a formação dos óxidos, bem como na facilidade da inclusão de dopantes, bastando para isto a utilização de soluções dos sais dos metais aditivos. $\mathrm{O}$ método se baseia na formação de quelatos entre cátions dissolvidos em solução de um ácido hidroxicarboxílico (ácido cítrico, por exemplo) com um poli(álcool hidroxílico). Nitratos são preferidos pela relativamente fácil eliminação durante a calcinação. Os nitratos e o ácido cítrico são misturados com um poli(álcool hidroxílico), podendo ser etileno glicol ou di-etileno glicol, e agitado enquanto aquecido a temperaturas de $70{ }^{\circ} \mathrm{C}$ a $110{ }^{\circ} \mathrm{C}$ até solução completa dos componentes. Aquecimento a temperaturas moderadas (120 a $250{ }^{\circ} \mathrm{C}$ ) causa assim uma reação de condensação entre o ácido cítrico e o etileno glicol com a formação de moléculas de $\mathrm{H}_{2} \mathrm{O}$ como sub-produto. Durante esse aquecimento moderado há poliesterificação e a maior parte do excesso de $\mathrm{H}_{2} \mathrm{O}$ é eliminado, resultando numa resina polimérica.

Aidéiageral destemétodoédistribuirosátomosaleatoriamente pela estrutura do polímero. A calcinação da resina ao ar ou outros gases causa a quebra do polímero e a conseqüente estruturação a $400{ }^{\circ} \mathrm{C}$. Os cátions são então oxidados para formar os cristalitos das mistura dos óxidos de $500^{\circ} \mathrm{Ca} 900^{\circ} \mathrm{C}$. O processo é bastante complexo e uma série de variáveis experimentais afetam o produto final [4]. Nessas variáveis contamos as impurezas incluídas nos precursores, bem como as condições de pirólise e calcinação. Isto inclui a atmosfera utilizada, que pode favorecer oxidações e outros processos químicos, e a velocidade e tempo de aquecimento, que podem beneficiar um determinado fator cinético ou estado termodinâmico.

Contudo, o processo baseado na patente de Pechini é potencialmente interessante, pois o único tipo de ânion presente no momento da oxidação pode ser também pirolisado. Ademais, a presença da água no momento da cristalização dos 
óxidos pode ser controlada, o que é um grande desafio quando tratando dos métodos de precipitação em soluções aquosas. Por esses motivos, a atividade da água e de diferentes cátions e ânions durante a cristalização dos óxidos é possível de ser verificada sob condições controladas [4]. Outra característica que faz esse método interessante no estudo da cristalização e do crescimento cristalino com relação aos aditivos é que a formação dos óxidos é independente de suas solubilidades. Deste modo, a preparação de óxidos com concentrações variadas e podendo conter diferentes cátions e ânions torna-se possível e com produtos bastante homogêneos e de estequiometria precisa e conhecida.

A presença de $\mathrm{H}_{2} \mathrm{O}$ na atmosfera de síntese e na transformação de fase da alumina tem implicação fundamental na morfologia e polimorfismo do óxido e na ação dos aditivos no controle da microestrutura [5-8]. Uma comparação do efeito da rota de síntese na atividade do íon Ti como aditivo na alumina mostrou claramente esta diferença [9]. Utilizando o método de Pechini para síntese da alumina contendo $1 \%$ Ti foi observada a evolução de estrutura a partir da fase amorfa para fase $\mathrm{\kappa}-\mathrm{Al}_{2} \mathrm{O}_{3}$. A mesma composição sintetizada tanto pelo método de sol-gel como por co-precipitação em solução aquosa, calcinada na mesma temperatura, mostrou uma evolução para a fase $\gamma-\mathrm{Al}_{2} \mathrm{O}_{3}$. Não foi proposta relação entre este fenômeno e o fato de que uma diferença marcante entre os métodos é que no método Pechini a presença de $\mathrm{H}_{2} \mathrm{O}$ é desprezível [4]. Entretanto, observando o efeito da $\mathrm{H}_{2} \mathrm{O}$ na cristalização da alumina a partir da boemita [5] e na cristalização da $\gamma-\mathrm{Al}_{2} \mathrm{O}_{3}$ [10], pode-se derivar essa conclusão, fazendo necessário um controle do teor de $\mathrm{H}_{2} \mathrm{O}$ em todos os ensaios de síntese, o que é praticamente impossível quando tratando de co-precipitação em meio aquoso ou sol-gel.

$\mathrm{O}$ efeito da presença de água na atmosfera de transformação da alumina já foi alvo de alguns estudos. $\mathrm{O}$ efeito de $\mathrm{H}_{2} \mathrm{O}$ no sistema é descrito não apenas como diminuidor local da temperatura na superficie das partículas, que pode diminuir a velocidade de cristalização, mas como também modificador de fluxos difusionais $[5,8,11]$. No entanto, sabe-se ainda que moléculas de $\mathrm{H}_{2} \mathrm{O}$ sobre a superfície da alumina podem diminuir significativamente as energias das interfaces [12], o que pode gerar alguns problemas experimentais principalmente quando o objetivo do estudo é uma avaliação sistemática do efeito de aditivos nas energias do sistema. Isto porque as moléculas de $\mathrm{H}_{2} \mathrm{O}$ afetam diferentemente as energias de superfície de diferentes fases [13], podendo induzir a formação de um determinado polimorfo durante a síntese e diminuindo, ou ainda aumentando, a temperatura de transformação entre eles.

Neste trabalho foi avaliada a ação da água na cristalização e transformação de fase da alumina sintetizada pelo método Pechini. Foram estudados pós de $\mathrm{Al}_{2} \mathrm{O}_{3}$ livre de aditivos e contendo $\mathrm{Mn}$ e $\mathrm{Mg}$ sintetizados em atmosfera úmida e seca, e elaborada uma explicação baseada no efeito da $\mathrm{H}_{2} \mathrm{O}$ nas energias de cada fase.

\section{PROCEDIMENTO EXPERIMENTAL}

Para produção da alumina foi utilizado o método baseado na patente de Pechini [4]. Pechini [3] propôs uma via de preparação química de óxidos que limita a adição de contra íons inorgânicos e permite a cristalização lenta das partículas. O precursor polimérico foi preparado pela adição de $\mathrm{Al}\left(\mathrm{NO}_{3}\right)_{3}$ anidro P.A. (Synth, $>98 \%$ ) à uma solução de ácido cítrico anidro P.A. (Synth, $>99,5 \%$ ) e etileno glicol P.A. (Synth) na proporção $25 \%, 45 \%$ e $30 \%$ em massa, respectivamente. Essa composição foi determinada de modo a maximizar a oxigenação do sistema [4]. Seguiuse um processo de solubilização total dos componentes a $\sim 70^{\circ} \mathrm{C}$ e polimerização a $\sim 120^{\circ} \mathrm{C}$. A resina polimérica obtida foi então calcinada a $400{ }^{\circ} \mathrm{C}$ por $4 \mathrm{~h}$ para decomposição térmica do polímero [4]. Após desaglomeração em almofariz de ágata, o material obtido foi calcinado novamente a $650{ }^{\circ} \mathrm{C}$ por $15 \mathrm{~h}$ para oxidação dos cátions e formação dos cristalitos. Esta última oxidação foi feita em um forno tubular com controle da atmosfera. $O$ controle para obtenção de atmosfera de ar seco foi feito fazendo passar ar sintético superseco com um fluxo de $60 \mathrm{~mL} \mathrm{~min}{ }^{-1}$ pelo tubo cerâmico do forno de calcinação. A presença de $\mathrm{H}_{2} \mathrm{O}$ na atmosfera de queima foi controlada fazendo passar um fluxo também de $60 \mathrm{~mL} \mathrm{~min}{ }^{-1}$ por um recipiente com quantidade fixa de $\mathrm{H}_{2} \mathrm{O}$ a $25^{\circ} \mathrm{C}$ antes de ser injetado no tubo do forno. $\mathrm{O}$ sistema foi completamente vedado em ambos os casos e um borbulhador na extremidade de saída de ar garantiu uma pressão positiva e constante no interior do tubo.

Aluminas contendo diferentes concentrações dos aditivos $\mathrm{Mn}$ e $\mathrm{Mg}$ foram preparadas utilizando como precursores o carbonato de manganês (II) P.A. (Alfa Aesar, 99,9\%) e óxido de magnésio (Alfa Aesar, 99,95\%). Soluções dos sais precursores em concentrações calculadas para atingir 5 e $10 \mathrm{~mol} \% \mathrm{Mn}$ e 3 e $5 \mathrm{~mol} \% \mathrm{Mg}$ foram adicionadas à resina na etapa anterior a decomposição térmica, garantindo assim boa homogeneidade de distribuição química do aditivo no pó de $\mathrm{Al}_{2} \mathrm{O}_{3}$.

Pós de $\mathrm{Al}_{2} \mathrm{O}_{3}$ livre de aditivos e contendo $\mathrm{Mn}$ ou $\mathrm{Mg}$ como aditivos foram sintetizados sob cada atmosfera e então submetidos à análise térmica diferencial (ATD) a $10{ }^{\circ} \mathrm{C} \mathrm{min}{ }^{-1}$ com atmosfera seca para avaliar as diferenças entre os pós produzidos pelos dois procedimentos.

\section{RESULTADOS E DISCUSSÃO}

O efeito da umidade na atmosfera de síntese foi estudado inicialmente avaliando a curva de ATD (em atmosfera seca) para amostras de mesma composição sintetizadas nas duas atmosferas (seca e úmida). Não houve variação significativa entre as curvas obtidas para $\mathrm{Al}_{2} \mathrm{O}_{3}$ livre de aditivos sintetizados sob as duas atmosferas. No entanto, para os materiais contendo os aditivos, a atmosfera de síntese mostrou-se determinante no produto final e na evolução da curva de ATD. A Fig. 1 mostra os gráficos de ATD para amostras de $\mathrm{Al}_{2} \mathrm{O}_{3}$ contendo $10 \mathrm{~mol} \% \mathrm{Mn}$ produzidas nas duas situações.

Para $\mathrm{Al}_{2} \mathrm{O}_{3}$ sintetizada sob atmosfera úmida há a presença dos dois picos exotérmicos característicos atribuídos à transformação de fase de amorfa para $\gamma-\mathrm{Al}_{2} \mathrm{O}_{3} \mathrm{a} \sim 877{ }^{\circ} \mathrm{C}$ 


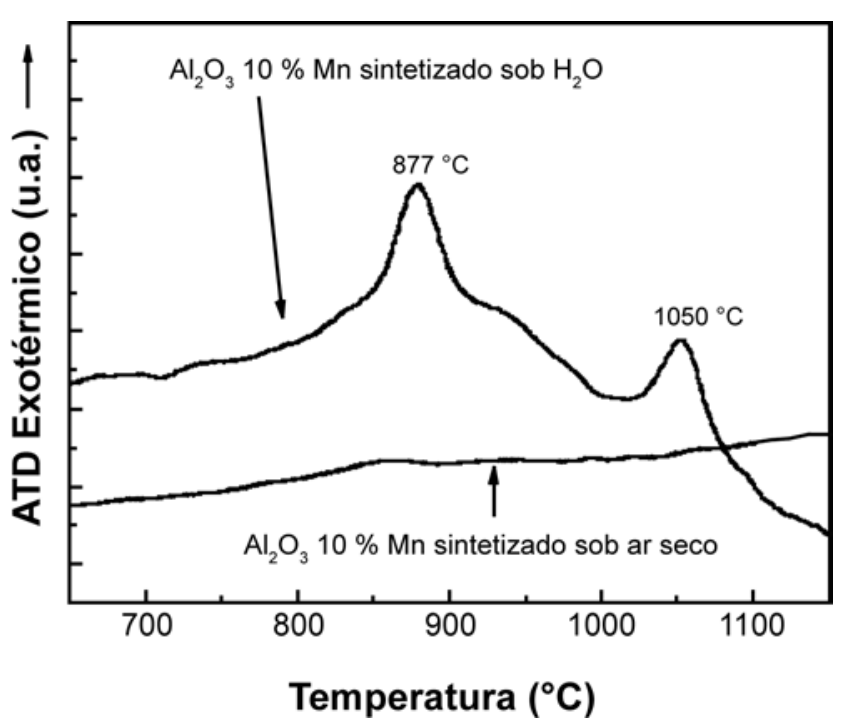

Figura 1: Curva de ATD das amostras de $\mathrm{Al}_{2} \mathrm{O}_{3}$ contendo $10 \mathrm{~mol} \%$ Mn sintetizadas pelo método dos precursores poliméricos em atmosfera seca e contendo $\mathrm{H}_{2} \mathrm{O}$.

[Figure 1: DTA of $\mathrm{Al}_{2} \mathrm{O}_{3}$ samples containing $10 \mathrm{~mol} \% \mathrm{Mn}$ synthesized by the polymeric precursor method under dry atmosphere (flat lower graph) and humidified atmosphere (upper graph).]

e de $\gamma-\mathrm{Al}_{2} \mathrm{O}_{3}$ para $\alpha-\mathrm{Al}_{2} \mathrm{O}_{3} \mathrm{a} \sim 1050{ }^{\circ} \mathrm{C}$. Quando não há $\mathrm{H}_{2} \mathrm{O}$ na atmosfera de síntese, os picos referentes a estas duas fases não estão presentes. Este fato é incoerente com uma atribuição apenas difusional da função da água na atmosfera de síntese. Isto é, a ação da água na cristalização e transformação de fase é sempre descrita como relacionada

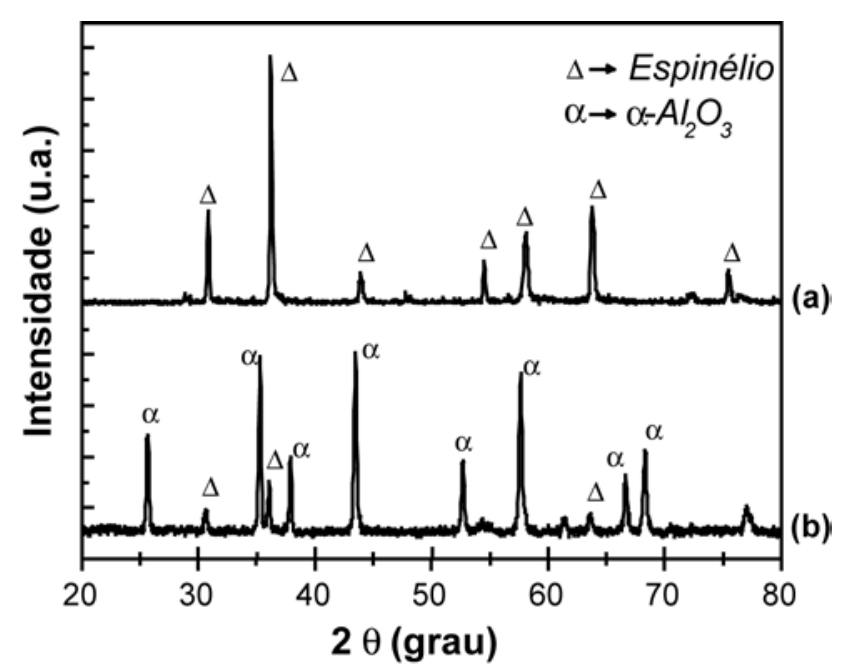

Figura 2: Difração de raios X das amostras contendo $10 \mathrm{~mol} \% \mathrm{Mn}$ sintetizadas em ar seco (a) e contendo $\mathrm{H}_{2} \mathrm{O}$ (b) após ensaio de ATD em atmosfera seca. A legenda das fases é mostrada no gráfico.

[Figure 2: X-ray diffraction patterns of samples containing $10 \mathrm{~mol} \%$ Mn synthesized under dry air atmosphere (a) and under humidified atmosphere (b) after DTA measurements under dry atmosphere. $\Delta$ represents spinel phase and $\alpha$ represents $\alpha-\mathrm{Al}_{2} \mathrm{O}_{3}$.] apenas ao incremento no processo de difusão e diminuição das energias de ativação [8]. Entretanto, a Fig. 2 mostra a difração de raios $\mathrm{X}$ dos pós obtidos após a ATD e indica fases diferentes para as duas atmosferas de síntese.

A presença de $\alpha-\mathrm{Al}_{2} \mathrm{O}_{3}$ é observada para o material sintetizado em atmosfera úmida com uma segunda fase identificada como um aluminato de manganês. A presença desta fase secundária está relacionada à diferença de solubilidade do $\mathrm{Mn}$ dentro a rede de $\gamma-\mathrm{Al}_{2} \mathrm{O}_{3}$ e de $\alpha-\mathrm{Al}_{2} \mathrm{O}_{3}$ após a transformação. Isto é, devido a estrutura espinélio com vacâncias da $\gamma-\mathrm{Al}_{2} \mathrm{O}_{3}[14]$ e $\mathrm{a}$ inclinação do íon $\mathrm{Mn}$ à formação de espinélio, espera-se uma solubilização considerável deste íon na $\gamma-\mathrm{Al}_{2} \mathrm{O}_{3}$. Quando a fase é $\alpha-\mathrm{Al}_{2} \mathrm{O}_{3}$, a solubilização é consideravelmente menor desde que a estrutura é mais compacta [15]. Deste modo há segregação do íon após a transformação que favorece a nucleação de uma fase secundária [16].

Para $\mathrm{Al}_{2} \mathrm{O}_{3} 10 \mathrm{~mol} \% \mathrm{Mn}$ sintetizada sob atmosfera seca não é observada a fase $\alpha-\mathrm{Al}_{2} \mathrm{O}_{3}$ após a ATD. Apenas um aluminato de manganês com estrutura semelhante à fase espinélio galaxita pôde ser observado. A ausência dos picos de cristalização de $\gamma-\mathrm{Al}_{2} \mathrm{O}_{3}$ e transformação para $\alpha-\mathrm{Al}_{2} \mathrm{O}_{3}$ na ATD é coerente com os dados de difração de raios $\mathrm{X}$ e indica a cristalização do espinélio em temperaturas mais baixas, assim como para a formação de $\beta$-aluminas $[17,18]$. Para a $\mathrm{Al}_{2} \mathrm{O}_{3}$ contendo $5 \mathrm{~mol} \% \mathrm{Mn}$ é observado efeito semelhante (Fig. 3), mas mesmo para atmosfera seca observa-se a presença de $\alpha-\mathrm{Al}_{2} \mathrm{O}_{3}$, mas em uma proporção muito menor que a observada na síntese em atmosfera úmida.

Observando os diferentes estados de oxidação possíveis para o íon $\mathrm{Mn}$ [19], poder-se-ia supor que a presença ou não de $\mathrm{H}_{2} \mathrm{O}$ induz diferentes estados que seriam responsáveis pelas diferentes

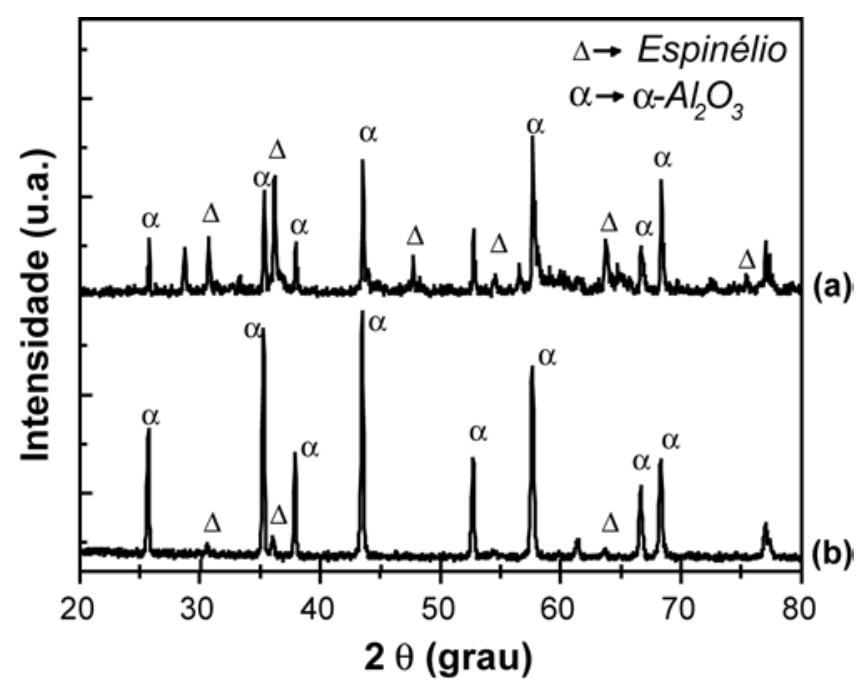

Figura 3: Difração de raios $\mathrm{X}$ das amostras contendo $5 \mathrm{~mol} \% \mathrm{Mn}$ sintetizadas em ar seco (a) e contendo $\mathrm{H}_{2} \mathrm{O}$ (b) após ensaio de ATD em atmosfera seca. A legenda das fases é mostrada no gráfico.

[Figure 3: X-ray diffraction patterns of the samples containing 5 mol\% Mn synthesized under dry air (a) and under humidified atmosphere (b) after DTA measurements under dry air. $\Delta$ represents spinel phase and $\alpha$ represents $\alpha-\mathrm{Al}_{2} \mathrm{O}_{3}$.] 
fases presentes em cada atmosfera. No entanto, o gráfico da Fig. 4 mostra que um efeito similar ao observado para os pós contendo Mn está presente nos pós de alumina contendo Mg como aditivo. Na Fig. 4 são mostrados os gráficos de ATD (em atmosfera seca) das amostras de $\mathrm{Al}_{2} \mathrm{O}_{3}$ contendo $5 \mathrm{~mol} \% \mathrm{Mg}$ sintetizadas sob atmosfera úmida e seca. Assim como para o caso do Mn, há omissão dos picos exotérmicos de cristalização de gama e transformação para alfa, indicando um fenômeno semelhante.

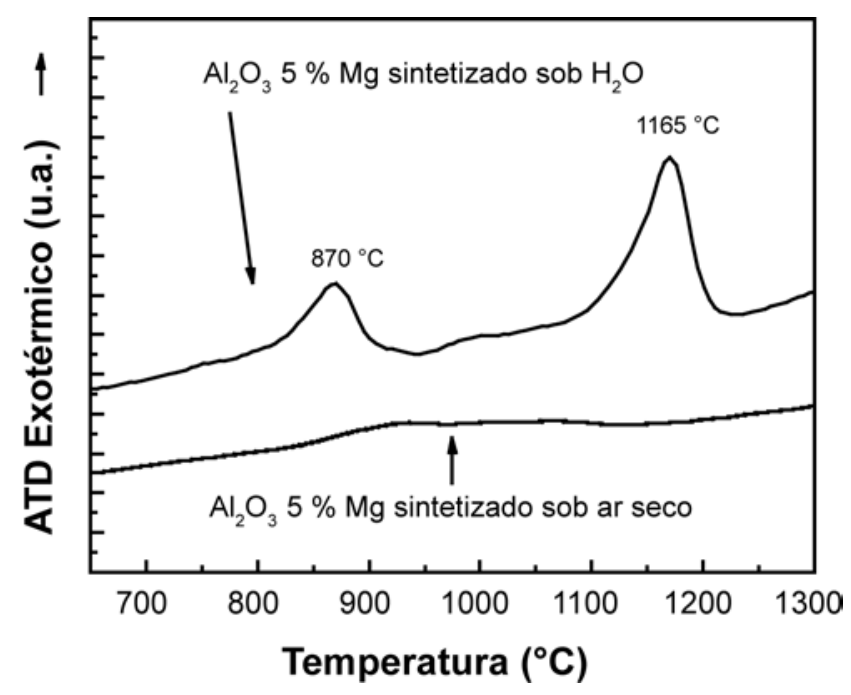

Figura 4: Curva de ATD das amostras de $\mathrm{Al}_{2} \mathrm{O}_{3}$ contendo $5 \mathrm{~mol} \%$ $\mathrm{Mg}$ sintetizadas pelo método dos precursores poliméricos em atmosfera seca e contendo $\mathrm{H}_{2} \mathrm{O}$.

[Figure 4: DTA data obtained for $\mathrm{Al}_{2} \mathrm{O}_{3}$ samples containing $5 \mathrm{~mol} \% \mathrm{Mg}$ synthesized by the polymeric precursor method under dry atmosphere (flat lower graph) and under humidified air (upper graph).]

Os gráficos de difração de raios $\mathrm{X}$ das amostras mostrados na Fig. 5 mostram a formação de uma fase espinélio também para $5 \mathrm{~mol} \% \mathrm{Mg}$ sintetizado sob atmosfera seca após a ATD. Para a amostra sintetizada na presença de $\mathrm{H}_{2} \mathrm{O}$ há apenas pequenos traços da formação do espinélio e a fase dominante é $\alpha-\mathrm{Al}_{2} \mathrm{O}_{3}$. Para $\mathrm{Al}_{2} \mathrm{O}_{3}$ contendo $3 \mathrm{~mol} \%$ de $\mathrm{Mg}$ comportamento similar foi observado, mas em ambas as atmosferas $\alpha-\mathrm{Al}_{2} \mathrm{O}_{3}$ foi detectada (Fig. 6), mas em atmosfera seca a fase espinélio é preponderante.

$\mathrm{O}$ efeito semelhante tanto para as amostras contendo Mn e Mg desacredita uma teoria baseada no número de oxidação pois a valência do íon $\mathrm{Mg}$ é usualmente 2+ [19]. Desta forma, duas hipóteses podem ser introduzidas sobre a ação da $\mathrm{H}_{2} \mathrm{O}$ na síntese. A primeira é referente a influência das moléculas de $\mathrm{H}_{2} \mathrm{O}$ na superfície das fases envolvidas. Como descrito na literatura, existe uma ação significativa da energia de superfície na temperatura de transformação e também na estabilidade de fases da alumina [13]. Sabendo que a presença de $\mathrm{H}_{2} \mathrm{O}$ sobre uma superfície muda diferentemente as energias de estruturas e composições [13, 20-22] e que espinélios de $\mathrm{Mn}$ e $\mathrm{Mg}$ têm grande afinidade por moléculas de $\mathrm{H}_{2} \mathrm{O}$ [23], como resultado de uma elevada energia de adsorção, é coerente que as moléculas de $\mathrm{H}_{2} \mathrm{O}$ estabilizem a superfície destes espinélio, promovendo

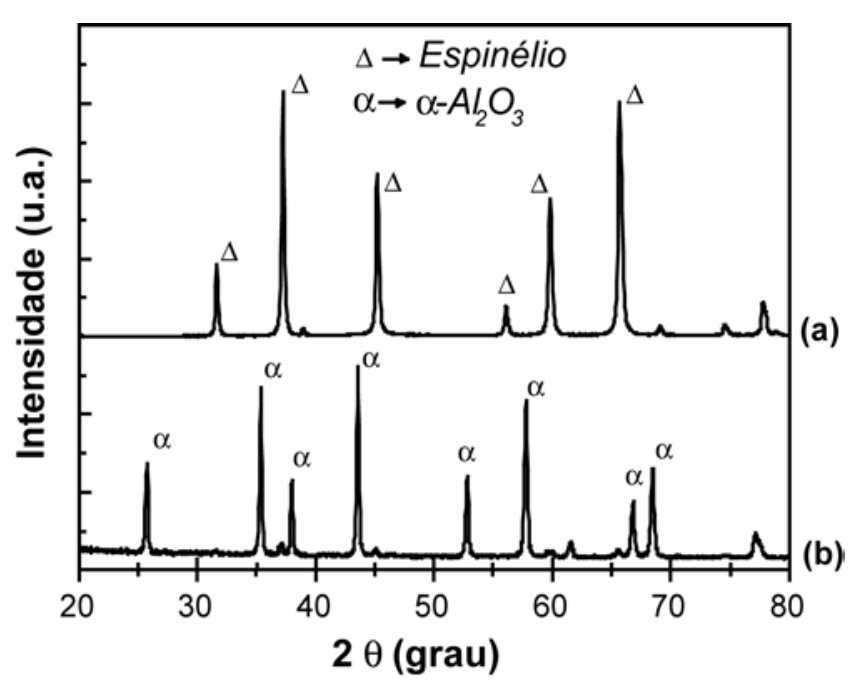

Figura 5: Difração de raios $\mathrm{X}$ das amostras contendo $5 \mathrm{~mol} \% \mathrm{Mg}$ sintetizadas em ar seco (a) e contendo $\mathrm{H}_{2} \mathrm{O}$ (b) após ensaio de DTA em atmosfera seca. A legenda das fases é mostrada no gráfico.

[Figure 5: X-ray diffraction patterns of the samples containing 5 mol\% Mg synthesized under dry air (a) and under humidified atmosphere (b) after DTA measurements under dry air. $\Delta$ represents spinel phase and $\alpha$ represents $\alpha-\mathrm{Al}_{2} \mathrm{O}_{3}$.]

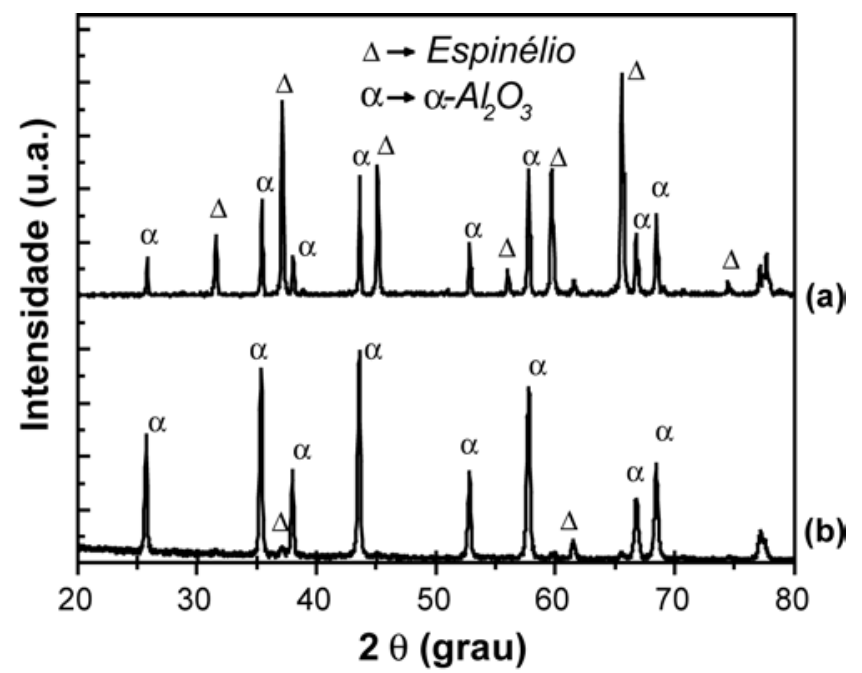

Figura 6: Difração de raios $\mathrm{X}$ das amostras contendo $3 \mathrm{~mol} \% \mathrm{Mg}$ sintetizadas em ar seco (a) e contendo $\mathrm{H}_{2} \mathrm{O}$ (b) após ensaio de DTA em atmosfera seca. A legenda das fases presentes é mostrada no gráfico.

[Figure 6: X-ray diffraction patterns of the samples containing 3 mol\% Mg synthesized under dry air (a) and under humidified atmosphere (b) after DTA measurements under dry air. $\Delta$ represents spinel phase and $\alpha$ represents $\alpha-\mathrm{Al}_{2} \mathrm{O}_{3}$.]

sua formação em detrimento à formação do espinélio defeituoso característico de $\gamma-\mathrm{Al}_{2} \mathrm{O}_{3}$.

A outra hipótese coerente é relacionada ao tipo de estrutura do $\gamma-\mathrm{Al}_{2} \mathrm{O}_{3}$. Embora amplamente utilizados, os dados disponíveis sobre a estrutura da $\gamma$-alumina ainda são bastante contraditórios. Grande parte da literatura estabelece a estrutura como tipo espinélio com defeito, 
sendo muito próxima da estrutura do espinélio $\mathrm{MgAl}_{2} \mathrm{O}_{4}$ $[11,14,15,24-26]$ com os íons $\mathrm{Al}^{3+}$ ocupando tanto posições catiônicas tetraédricas como octaédricas na estrutura cúbica compacta dos oxigênios [27]. No entanto, para satisfazer a estequiometria do óxido de alumínio, $2^{2} / 3$ dos 24 sítios catiônicos devem ser lacunas. A fórmula da $\gamma$-alumina é, portanto usualmente descrita como $\square_{2^{3 / 3}} \mathrm{Al}_{21^{\prime}, 3} \mathrm{O}_{32}\left(\mathrm{ou} \mathrm{Al}_{8 / 3} \mathrm{O}_{4}\right)$ onde $\square$ significa uma vacância [14]. Outra vertente, no entanto, sugere a presença de prótons na estabilização da estrutura. Foi sugerido [28] que prótons poderiam residir nas lacunas de cátions presentes na estrutura de gama alumina. Tal situação foi mais esclarecida após a proposta de que a subrede de oxigênio da $\gamma-\mathrm{Al}_{2} \mathrm{O}_{3}$ é sempre completa, mas falta uma fração de átomos de alumínio na estrutura, sendo compensada pela presença de $\mathrm{H}+$ [29]. Deste modo, foi proposta uma nova fórmula $\mathrm{H}_{3 \mathrm{~m}} \mathrm{Al}_{2-\mathrm{m}} \mathrm{O}_{3}$, onde $m=2 n /(n+3)$, sendo $n$ o número de hidratação baseado em cálculos teóricos de densidade.

De certo a presença de $\mathrm{H}_{2} \mathrm{O}$ na atmosfera de síntese na etapa de cristalização do método Pechini forneceria prótons para incorporação na rede cristalina. Isto é consistente com a cristalização desta fase quando da síntese em atmosfera úmida e a cristalização de outra fase espinélio sob atmosfera seca, fortalecendo a hipótese de presença de $\mathrm{H}^{+}$na estrutura $\gamma-\mathrm{Al}_{2} \mathrm{O}_{3}$

\section{CONCLUSÃO}

Mostrou-se uma significativa dependência do processo de cristalização na presença de $\mathrm{H}_{2} \mathrm{O}$ na atmosfera de síntese. $\mathrm{O}$ método dos precursores poliméricos permitiu a síntese de alumina contendo $\mathrm{Mg}$ ou $\mathrm{Mn}$ como aditivos na ausência de $\mathrm{H}_{2} \mathrm{O}$ e sob atmosfera úmida. A evolução das fases foi disparate para as duas atmosferas, sendo um aluminato de magnésio (ou manganês) induzido pela ausência de $\mathrm{H}_{2} \mathrm{O}$ enquanto que a fase $\alpha-\mathrm{Al}_{2} \mathrm{O}_{3}$ surge na presença de $\mathrm{H}_{2} \mathrm{O}$. Foi sugerida uma atividade da $\mathrm{H}_{2} \mathrm{O}$ como doadora de prótons para manter a estabilidade do $\gamma-\mathrm{Al}_{2} \mathrm{O}_{3}$ durante a cristalização.

$\mathrm{O}$ trabalho ressalta assim a importância de um controle da atmosfera quando do estudo da cristalização da alumina, e indica o método dos precursores poliméricos como uma solução para o controle de $\mathrm{H}_{2} \mathrm{O}$, desde que em métodos mais comum de síntese tais como sol-gel e precipitação a presença de $\mathrm{H}_{2} \mathrm{O}$ é inerente ao processo.

\section{AGRADECIMENTOS}

CAPES (Coordenação de Aperfeiçoamento de Pessoal de Nível Superior) e FAPESP (Fundação de Amparo à Pesquisa do Estado de S. Paulo), Processos 96/09604-9, 99/10798-0 e 01/10053-7, pelo apoio financeiro.

\section{REFERÊNCIAS}

[1] D. Gouvêa, R. L. Villalobos, J. D. T. Capocchi, Mater. Sci. Forum. 299, 3 (1999) 91.

[2] M. I. B. Bernardi, S. C. L. Crispim, A. P. Maciel, A. G.
Souza, M. M. Conceição, E. R. Leite, E. Longo, J. Therm. Anal. Calorim. 75, 2 (2004) 475.

[3] M. Pechini, "Method of preparing lead and alkaline earth titanates and niobates and coating method using the same form a capacitor," US Patent (1967).

[4] P. A. Lessing, Am. Ceram. Soc. Bull. 68, 5 (1989) 1002.

[5] Z. Hrabe, S. Komarneni, L. Pach, R. Roy, J. Mater. Res. 7, 2 (1992) 444.

[6] R. Petrovic, S. Milonjic, V. Jokanovic, L. KosticGvozdenovic, I. Petrovic-Prelevic, D. Janackovic, Powder Technol. 133, 1-3 (2003) 185.

[7] T. F. Baumann, A. E. Gash, S. C. Chinn, A. M. Sawvel, R. S. Maxwell. J. H. Satcher, Chem. Mater. 17, 2 (2005) 395.

[8] R. B. Bagwell, G. L. Messing, J. Am. Ceram. Soc. 82, 4 (1999) 825.

[9] T. Hernandez, M. C. Bautista, J. Eur. Ceram. Soc. 25 (2005) 663.

[10] H. C. Lee, H. J. Kim, C. H. Rhee, K. H. Lee, J. S. Lee, S. H. Chung, Micropor. Mesopor. Mat. 79 (2005) 61.

[11] I. Levin, D. Brandon, J. Am. Ceram. Soc. 81, 8 (1998) 1995.

[12] N. H. de Leeuw, S. C. Parker, J. Am. Ceram. Soc. 82, 11 (1999) 3209.

[13] J. M. McHale, A. Auroux, A. J. Perrotta, A. Navrotsky, Science 277, 5327 (1997) 788.

[14] R. S. Zhou, R. L. Snyder, Acta Crystall. B-Struct. 47 (1991) 617.

[15] E. Dörre, Alumina: Processing, properties, and applications, Springer-Verlag, Berlin (1984).

[16] R. H. R. Castro, D. Gouvêa, Cerâmica 49 (2003) 55.

[17] F. R. Feret, D. Roy, C. Boulanger, Spectrochim. Acta B 55, 7 (2000) 1051.

[18] D. R. Treadwell, A. C. Sutorik, S. S. Neo, R. M. Laine, R. C. Svedberg, "Synthesis of beta"-alumina polymer precursor and ultrafine beta"-alumina composition powders", Synthesis and Characterization of Advanced Materials, vol. 681 (1998) pp. 146.

[19] D. F. Shriver, P. W. Atckins, C. H. Langford, "Inorganic chemistry," Oxford University Press, EUA (1994).

[20] M. L. Gonzalez-Martin, L. Labajos-Broncano, B. Janczuk, J. M. Bruque, J. Mater. Sci. 34, 23 (1999) 5923.

[21] Z. Lodziana, J. K. Norskov and P. Stoltze, J. Chem. Phys. 118, 24 (2003) 11179.

[22] A. Navrotsky, Abstr. Pap. Am. Chem. S. 225 (2003) U939. [23] I. V. Vinnik, V. S. Zenkov, M. M. Sirotyuk, L. M. Koval'skii, I. V. Uvarova, Powder Metall. Met. C+ 37, 7-8 (1998) 382.

[24] G. Gutierrez, A. Taga, B. Johansson, Phys. Rev. B 65, 1 (2002). [25]H. F. Stumpf, A. S. Russel, J. W. Newsome, Ind. Eng. Chem. 42, 7 (1949) 1298.

[26] K. Wefers, C. Misra, Alcoa Technical Paper n. 19, revised (1987).

[27] G. N. Kryukova, D. O. Klenov, A. S. Ivanova, S. V. Tsybulya, J. Eur. Ceram. Soc. 20, 8 (2000) 1187.

[28] D. A. Dowden, Chem Ind-London 20 (1949) 320.

[29] K. Sohlberg, S. J. Pennycook, S. T. Pantelides, J. Am. Chem. Soc. 121, 33 (1999) 7493.

(Rec. 26/05/2005, Ac. 30/09/2005) 